\title{
LA COMPLEJIDAD QUÍMICA DE LAS GASOLINAS DE AUTOMOCIÓN
}

\author{
The chemical complexity of automotive gasolines
}

\author{
Carlos José Boluda a Mariana Macías ${ }^{\mathrm{b}}$ y Joaquín González \\ MARRERO $^{\mathrm{b}}$
}

Recibido: 21/10/19 - Aprobado: 16/12/19

Cómo citar: Boluda, C. J., Macías, M., \& González Marrero, J. (2019). La complejidad química de las gasolinas de automoción . Ciencia, Ingenierias Y Aplicaciones, 2(2), 51-79. Doi: https://doi.org/10.22206/cyap.2019.v2i2. pp51-79

\section{Resumen}

Utilizadas principalmente como combustibles en motores de combustión interna, las gasolinas son mezclas complejas de hidrocarburos que incluyen en su composición distintos aditivos para mejorar su estabilidad y prestaciones. Su composición quimica es no solo diversa, sino también variable, dependiendo del crudo de procedencia, del proceso de obtención y de las normativas existentes. Estas fijan el contenido máximo permitido de determinados compuestos que, por su toxicidad, deben quedar limitados. Las gasolinas se obtienen por destilación fraccionada del petróleo y también a partir de fracciones más pesadas del mismo, mediante craqueo térmico o catalitico, procesos que junto al reformado catalítico van a tener una incidencia directa en su composición. El objetivo de esta revisión es abordar la compleja composición que presentan las gasolinas, asi como algunos procesos básicos del refinado de petróleo. Estos procesos determinan en buena medida la naturaleza quimica de los componentes de este combustible, algunos de los cuales se relacionan con la emisión de sustancias nocivas para la salud y el medio ambiente. De esta forma, proporcionamos un contexto más exacto sobre los agentes contaminantes de la gasolina, a la vez que ponemos de manifiesto la necesidad de contar con normativas avanzadas que regulen la composición de los combustibles.

Palabras clave: : gasolina; octanaje; craqueo térmico; craqueo catalítico;

\footnotetext{
a Área de Ciencias Básicas y Ambientales, Instituto Tecnológico de Santo Domingo (INTEC), Av. de Los Próceres 49, Los Jardines del Norte 10602, Santo Domingo, República Dominicana Correo-e: carlos.boluda@intec.edu.do

b Instituto Politécnico Nacional, Unidad Profesional Interdisciplinaria de Ingeniería Campus Guanajuato, Av. Mineral de Valenciana, No. 200, Col. Fracc. Industrial Puerto Interior, C.P. 36275 Silao de la Victoria, Guanajuato, México. Correo-e: mmacias@ipn.mx, jgonzalezm@ipn.mx
} 
reformado catalítico.

\begin{abstract}
Used mainly as fuels in internal combustion engines, gasolines are complex mixtures of hydrocarbons that include in their composition different additives that improve their stability and performance. Its chemical composition is not only diverse but also variable, depending on the crude oil origin, the obtaining process and the existing regulations, which fix the maximum allowed content of certain compounds that should be limited due to their toxicity. Gasolines are obtained by fractional distillation of petroleum and also from heavier fractions of petroleum by thermal or catalytic cracking, processes that together with catalytic reforming will have a direct impact on their composition. The objective of this review is to address the complex composition of gasoline, as well as some basic processes of oil refining, which largely determine its composition and which are closely related to the emission of polluting substances. In this way, we provide a more accurate context on the pollutants of gasoline and its impact on the environment, while we highlight the need for advanced regulations to regulate the composition of fuels.
\end{abstract}

Keywords: gasoline; octane index; thermal cracking; catalytic cracking; catalytic reforming. 


\section{Introducción}

El petróleo es una sustancia de carácter oleaginoso que incluye, a temperatura ambiente, componentes sólidos, líquidos y gaseosos. Su color varía desde el amarillo o rojo pardo hasta el negro, que le da su aspecto más característico. Está compuesto por una mezcla diversa de hidrocarburos que, en estado natural, se encuentra en yacimientos subterráneos de los estratos superiores de la corteza terrestre; su proceso de refinado, mediante una serie de transformaciones químicas y físicas, proporciona una variedad de derivados de gran importancia industrial como la gasolina o el queroseno. Según el peso molecular de los hidrocarburos obtenidos durante el proceso de fraccionamiento del petróleo, se pueden diferenciar los siguientes derivados: gases de refinería, gases licuados de petróleo (propano y butano), naftas (pesadas y ligeras), querosenos, petróleos lampantes, combustibles de aviación, gasóleos de automoción y de calefacción, fuelóleos pesados industriales, aceites (ligeros y pesados), parafinas y ceras, asfalto y coque (Gary \& Handwerk, 1980).

Entre los derivados de petróleo, las gasolinas están entre los más utilizados. Se trata de combustibles líquidos usados en motores de combustión interna, compuestos por hidrocarburos con un número de carbonos comprendido entre 5 y 12 , que se obtienen por destilación fraccionada del petróleo con un intervalo de ebullición comprendido entre 30 y $225^{\circ} \mathrm{C}$ (Wiedemann, d'Avila \& Azevedo, 2005; Kinsara \& Demirbas, 2016). Pese a que el rango de pesos moleculares está acotado y a que, por definición, los hidrocarburos están compuestos únicamente por carbono e hidrógeno, se trata de mezclas complejas que pueden estar formadas por más de quinientos compuestos químicos diferentes, algunos de ellos tóxicos. Este hecho se pone claramente de manifiesto cuando estudiamos estos combustibles mediante diferentes técnicas analíticas como cromatografía de gases o espectroscopia de infrarrojos (Brudzewski et al., 2006; Lee, Lee \& Hwang, 2018; Vempatapu \& Kanaujia, 2017). 
La complejidad química de las gasolinas se debe, en parte, a la elevada diversidad estructural de sus componentes, dado que pueden estar presentes casi todos los isómeros posibles de los hidrocarburos que tienen entre $4 \mathrm{y}$ 12 átomos de carbono. Así, a los múltiples isómeros constitucionales que presentan sus hidrocarburos alifáticos saturados, lineales, ramificados y cíclicos, hay que ańadir los compuestos aromáticos y una fracción menos importante de olefinas. No obstante, pese a esta diversidad potencial, la fracción mayoritaria del combustible está representada por un grupo mucho más reducido de compuestos (Ritter, 2005).

Por una parte, está perfectamente establecido que la composición de las gasolinas está directamente vinculada a las emisiones del motor (Harley et al., 2006; Schifter et al., 2013), habiéndose realizado varios estudios exhaustivos para investigar el impacto de la composición de la gasolina en las emisiones de los vehículos. Estos incluyen, por ejemplo, el Programa de investigación para la mejora de la calidad del aire / aceite del automóvil (AQIRP) llevado a cabo a principios de la década de 1990, así como estudios realizados por la Agencia de Protección Ambiental de los Estados Unidos (Karavalakis et al., 2015). Por otra parte, estudios más recientes han evaluado el efecto de los compuestos oxigenados como el etanol en la volatilidad de la gasolina y las emisiones del motor, así como la influencia del contenido de olefinas en las emisiones de escape. Todas estas investigaciones nos ha permitido una mejor comprensión de la relación entre las formulaciones de gasolina y las emisión de contaminantes como los de óxidos de nitrógeno (NOx) o los compuestos orgánicos volátiles (Durbin et al., 2007; Hajbabaei et al., 2013).

Además de estructuralmente diversas, las gasolinas también son variables en su composición, dado que esta va a depender de factores como el tipo de crudo del cual se ha obtenido, del proceso de obtención en las refinerías y de las normativas vigentes de cada país (Re-Poppi et al., 2009). Por ello, las gasolinas, al igual que otros combustibles de automoción, deben cumplir con las especificaciones de los fabricantes de vehículos, cuyas orientaciones se recogen en la Carta Mundial de Combustibles y con las normativas vigentes de cada país. Dichas normativas han evolucionado 
a lo largo de las últimas décadas, aumentando notablemente los niveles de exigencia, con el objeto de proteger la salud de la población y el medio ambiente (World Wide Fuel Charter, 2013).

\section{Caracterización físico-química de las gasolinas}

\section{1. Índice de octanos}

Con el objeto de minimizar las emisiones de sustancias nocivas para la salud y el medio ambiente, así como cumplir con las especificaciones de los fabricantes de automóviles, las normativas de los distintos países establecen valores máximos y/o mínimos para una serie de parámetros físico-químicos que deben cumplir los combustibles de automoción. En este sentido resultan especialmente restrictivas las normativas de los países desarrollados, como Estados Unidos, Japón, Australia o los que conforman la Unión Europea.

Las especificaciones para las gasolinas incluyen la densidad, la presión de vapor, la curva de destilación, el índice de octanos y el contenido en determinados compuestos como benceno, aromáticos totales, olefinas, compuestos oxigenados y sulfurados. Algunos de estos parámetros, como es el caso del índice de octanos, se relacionan con el correcto funcionamiento de los motores y por tanto están en relación con las especificaciones técnicas de los fabricantes de vehículos. Otros parámetros en cambio, como el contenido en benceno y aromáticos totales, se relacionan además con la protección de la salud y el medio ambiente.

El índice de octanos u octanaje es una de las especificaciones más importantes de una gasolina, depende de su composición química y representa una medida de la resistencia a la autoignición del combustible. La detonación prematura de la gasolina, antes de que la produzca la chispa entre los electrodos de la bujía, genera frentes de llama con direcciones opuestas que frenan el movimiento ascendente del pistón. Este problema conocido como "picado de bielas" se manifiesta durante el funcionamiento del motor con un sonido característico, que es descrito como 
"cascabeleo", "golpeteo", "martilleo", "pistoneo" o "knocking" en la literatura en lengua inglesa. La necesidad de una capacidad antidetonante mínima es especialmente relevante en motores de gasolina con una elevada relación de compresión, y no satisfacer este requerimiento puede provocar la autoignición del combustible. Esto último se traduce en pérdida de eficiencia del motor y aumenta la probabilidad de dańos mecánicos severos (Demirbas et al., 2015). La exigencia sobre la capacidad antidetonante de las gasolinas ha ido aumentando paralelamente al desarrollo de la industria automovilística. Así, por ejemplo, un automóvil en la década de 1920 tenía una relación de compresión de 4.4:1 que le permitía emplear gasolina de tan solo 55 octanos, generando una potencia de 14 caballos de vapor (HP) por litro del cilindro. Estas exigencias pueden ser perfectamente satisfechas por las gasolinas de destilación directa, que poseen un octanaje de aproximadamente 70 . Con el primer motor V-8 presentado por Ford en 1932 comenzó el desarrollo de motores de alto rendimiento y ya en la década de 1960 el incremento en la relación de compresión hasta 9.5:1, permitía generar $48 \mathrm{HP}$ por litro, lo que requería gasolinas con un octanaje en torno a 95 (Schobert, 1990). Hoy en día los motores de los automóviles están provistos de sensores de detonación que les permiten operar con combustibles de bajo octanaje, pero a costa de incrementar el consumo de combustible y perder potencia, lo que tampoco garantiza la ausencia de autodetonaciones (World Wide Fuel Charter, 2013).

El octanaje de las gasolinas se determina experimentalmente en un motor monocilíndrico de laboratorio con relación de compresión variable en el que se va incrementando la compresión hasta que se produce la autoinflamación del combustible. Luego se compara el resultado con una mezcla binaria de isooctano y $n$-heptano de proporciones conocidas. El isooctano tiene unas propiedades antidetonantes excelentes y se le asigna arbitrariamente un valor de octanaje de 100 , mientras que el $n$-heptano tiene un comportamiento antidetonante muy bajo, por lo que se le asigna el valor cero. Se construye así una escala relativa, que tiene como extremos a ambos hidrocarburos, y que nos permite evaluar por comparación la 
capacidad antidetonante de las gasolinas. De esta manera, que una gasolina sea de 95 octanos significa que se comporta, en cuanto a su capacidad antidetonante, como una mezcla de 95 partes de isooctano y 5 de $n$-heptano (Perdih \& Perdih, 2006). El octanaje de las gasolinas puede determinarse bajo condiciones diferentes que producen resultados distintos. Por una parte, se pueden simular condiciones de conducción suaves, con una exigencia moderada para el motor, obteniéndose así el denominado Número de Octano de Investigación (Research Octane Number, RON). Por otra parte, sometiendo al motor a mayor carga obtendremos el Número de Octano del Motor (Motor Octane Number, MON). Los valores del RON son mayores que los del MON, no debiendo ser la diferencia mayor de 10 unidades. Para propósitos de comercialización y distribución de las gasolinas, los productores determinan el octanaje comercial, como el promedio de los números de octano de investigación (RON) y el octano del motor (MON), lo que define el conocido anti-nock index (AKI) (World Wide Fuel Charter, 2013). Se puede calcular de la siguiente forma:

$$
\text { Número de octano comercial }=\mathrm{AKI}=\frac{\mathrm{RON}+\mathrm{MON}}{2}
$$

La capacidad antidetonante de los hidrocarburos se relaciona con su estructura química, de manera que en el caso de los alcanos se observa que las isoparafinas tienen un mejor comportamiento que los n-alcanos. El índice de octanos depende también del peso molecular, del grado de ramificación, de la posición de dichas ramificaciones y de su separación. La dependencia entre la resistencia a la autoignición de las parafinas y su estructura se describe mediante un conjunto de reglas empíricas que establecen, por ejemplo, que el índice de octanos aumenta con el grado de ramificación y con una posición más central de las mismas. Por otra parte, los hidrocarburos aromáticos poseen índices de octano elevados, a menudo mayores de 100 (tabla 1). Por esta razón los hidrocarburos aromáticos se emplean como potenciadores del octanaje de las gasolinas (Demirbas et al., 2015; Perdih \& Perdih, 2006). 
Tabla 1. Algunos constituyentes de las gasolinas y sus números RON y MON, en orden creciente a su número AKI (Demirbas et al., 2015)

\begin{tabular}{|c|c|c|c|c|c|}
\hline Entrada & Nombre & Estructura & RON & MON & AKI \\
\hline 1 & n-Heptano & & 0 & 0 & - \\
\hline 2 & n-Octano & & 0 & 0 & - \\
\hline 3 & $n$-Hexano & & 24.8 & 26.0 & 25.4 \\
\hline 4 & $n$-Pentano & & 61.8 & 63.2 & 62.5 \\
\hline 5 & 2-Hepteno & & 73.4 & 68.8 & 71.1 \\
\hline 6 & 3-Hepteno & & 90.0 & 79.3 & 84.7 \\
\hline 7 & 3-Hexeno & & 94.0 & 80.1 & 87.1 \\
\hline 8 & 4-Octeno & & 94.6 & 80.6 & 87.6 \\
\hline 9 & 2-Penteno & & 87.8 & 87.8 & 87.8 \\
\hline 10 & Benceno & & 90.0 & 92.0 & 91.0 \\
\hline 11 & $\begin{array}{l}\text { 2,4,4-Trimetil-2- } \\
\text { penteno }\end{array}$ & & 103.5 & 86.2 & 94.9 \\
\hline 12 & tert-Butanol & & 103.0 & 91.0 & 97.0 \\
\hline 13 & Metanol & $\mathrm{CH}_{3} \mathrm{OH}$ & 108.7 & 88.6 & 98.7 \\
\hline 14 & Isooctano & & 100.0 & 100.0 & 100.0 \\
\hline 15 & Isopropanol & & 118.0 & 98.0 & 108.0 \\
\hline 16 & $o$-Xileno & & 103.0 & 120.0 & 111.5 \\
\hline 17 & Etilbenceno & & 107.0 & 124.0 & 115.5 \\
\hline
\end{tabular}




\begin{tabular}{|c|c|c|c|c|c|}
\hline Entrada & Nombre & Estructura & RON & MON & AKI \\
\hline 18 & Tolueno & & 112.0 & 124.0 & 118.0 \\
\hline 19 & Etanol & $\mathrm{CH}_{3} \mathrm{CH}_{2} \mathrm{OH}$ & 129.0 & 116.0 & 122.5 \\
\hline 20 & Propilbenceno & & 129.0 & 127.0 & 128.0 \\
\hline 21 & 1,3,5-Trimetilbenceno & & 137.0 & 124.0 & 130.5 \\
\hline 22 & m-xileno & & 124.0 & 145.0 & 134.5 \\
\hline 23 & $p$-Xileno & & 127.0 & 146.0 & 136.5 \\
\hline 24 & Gasolina Regular & - & 81.0 & 91.0 & 86 \\
\hline 25 & Gasolina Premiun & - & 85.0 & 95.0 & 90 \\
\hline
\end{tabular}

\subsection{La composición química de las gasolinas}

A nivel molecular, las gasolinas están compuestas por cientos de compuestos, que estructuralmente se pueden dividir en cuatro grupos principales: parafinas, olefinas, cicloparafinas y aromáticos. La fracción aromática de la gasolina esta principalmente representada por benceno, tolueno, etilbenceno y xilenos a los que suele hacerse referencia con el acrónimo BTEX (significa benceno, tolueno, etilbenceno y xilenos). También puede contener compuestos oxigenados, como alcoholes y éteres. Además, forman parte de la composición de este combustible diferentes aditivos, en concentraciones del orden de partes por millón (ppm), con funciones diversas como incrementar el octanaje, evitar la oxidación del combustible o mejorar la combustión. A continuación, describiremos brevemente cada uno de estos grupos de compuestos (Swick et al., 2014). 


\subsubsection{Parafinas y cicloparafinas}

Los alcanos son también conocidos como parafinas (del latín parum affinis), término que hace referencia a su falta de reactividad química, entendida como el limitado número de reacciones que caracteriza a los hidrocarburos saturados. Con una fórmula molecular $\mathrm{C}_{2} \mathrm{H}_{2 \mathrm{n}+2}$, las parafinas son el componente mayoritario de las gasolinas. Podemos subdividirlas en no ramificados ( $n$-alcanos) y parafinas ramificadas.

Dado que la resistencia a la autoginición es más elevada en los alcanos ramificados que en los no ramificados, la presencia de isoparafinas es deseable en las gasolinas. Se consiguen así valores más altos de octanaje, permitiendo que el combustible pueda ser empleado por motores de alta compresión. Los cicloalcanos o cicloparafinas, también presentes en la gasolina, son hidrocarburos de naturaleza cíclica y alifática por lo que también puede utilizarse el término alicíclico. Algunas gasolinas presentan más de un $40 \%$ de este tipo de hidrocarburos en su composición. Las gasolinas que provienen del campo petrolero de Midway (California) son especialmente ricas en ciclopentanos con un $41 \%$ de estos hidrocarburos mientras que procedentes del petróleo Conroe (Texas) es rica en ciclohexanos con un $42 \%$ (Curiale \& Frolov, 1998). Asimismo, en las gasolinas sometidas a procesos de reformado, encontramos frecuentemente ciclopentano, ciclohexano y sus derivados alquil-sustituidos (Ramadhan \& Al-Hyali, 1999).

\subsubsection{Hidrocarburos aromáticos}

Los hidrocarburos aromáticos presentes en las gasolinas incluyen principalmente la fracción BTEX, además de otros de mayor peso molecular como es el caso del propilbenceno y el 1,3,5-trimetilbenceno (mesitileno) (Demirbas et al., 2015). Esta representa una de las fracciones más pesadas de la gasolina, con un rango de ebullición comprendido entre los 40 y los $180^{\circ} \mathrm{C}$ (Ramadhan \& Al-Hyali, 1999). El crudo procedente de Extremo Oriente suele producir gasolinas con un contenido en aromáticos elevado (Curiale \& Frolov, 1998). 
Dado que los octanajes de los compuestos aromáticos se encuentran entre los más altos de aquellos hidrocarburos que forman parte de las gasolinas (tabla 1, entradas 10,16-18, 20-23), son componentes importantes de este combustible. Sin embargo, a pesar de que su restricción requiere de otras estrategias para alcanzar el octanaje apropiado con un costo mayor, a lo largo de las últimas décadas ha existido una clara tendencia internacional a limitar su presencia en los combustibles, debido a su toxicidad y a la relación demostrada entre la formulación exacta de las gasolinas y la calidad del aire (Australian Government, 2000). Así, por ejemplo, la Unión Europea fijó en el año 2000 un máximo en aromáticos totales del $42 \% \mathrm{v} / \mathrm{v}$, para luego reducirlo al $35 \%$ v/v en 2005 (Karavalakis et al., 2015). Aunque la normativa japonesa limita la cantidad de benceno al $1 \% \mathrm{v} / \mathrm{v}$ en sus gasolinas, no incluye límites para el total de compuestos aromáticos. Esto, porque los reguladores japoneses consideraron innecesaria esta limitación, dado que el contenido en aromáticos de sus gasolinas siempre oscila entre el 25 y el $30 \%(\mathrm{v} / \mathrm{v})$ y solo se acerca al $35 \%$ (valores máximos en Europa) en el caso de la gasolina Premium (Australian Government, 2014).

El caso del benceno merece especial atención dado su reconocida toxicidad (Yardley-Jones et al., 1991; Khalade et al., 2010). Este es un componente normal del petróleo que se genera también durante el reformado catalítico y se libera al medio ambiente mediante evaporación desde el combustible y emisión con los gases de escape de los vehículos (World Wide Fuel Charter, 2013). Además, también puede producirse benceno durante la combustión de otros compuestos aromáticos para luego ser liberado al medio ambiente. Esto explica por qué la reducción del contenido total en hidrocarburos aromáticos en las gasolinas tiene como consecuencia la disminución de la emisión de benceno en los gases de escape (World Wide Fuel Charter, 2013; Yao \& Tsai, 2013). Debido a su peligrosidad, es el único hidrocarburo aromático con una limitación específica en torno al $1 \% \mathrm{v} / \mathrm{v}$ en las normativas más avanzadas. Así, por ejemplo, la Unión Europea limitó el contenido máximo de benceno al $1 \%$, al igual que Canadá, que tiene la misma limitación desde julio de 1999, y Australia desde 2001. Estados Unidos fijó el límite para el contenido 
en benceno a un promedio de $0.95 \% \mathrm{v} / \mathrm{v}$ en enero de 1995. Japón introdujo una limitación del $5 \%$ v/v en 1996 y fue reducida al $1 \%$ en 2000 (Verma \& des Tombe, 2002).

Aunque no faltan referencias sobre la toxicidad del tolueno (Donald, Hooper \& Hopenhayn-Rich, 1991; Von Euler et al., 2000; Yang et al., 2010), es considerado menos tóxico que el benceno y debido a su elevado índice de octanos $(\mathrm{RON}=112.0 / \mathrm{MON}=124.0)$ (tabla 1, entrada 18), lo ha sustituido como potenciador del octanaje de las gasolinas. Ni la Carta Mundial del Combustible ni los países que poseen las especificaciones más avanzadas limitan específicamente el contenido en tolueno; no obstante, el contenido en tolueno queda indirectamente limitado debido a la restricción de aromáticos totales incluida en las normativas avanzadas.

Es evidente que todo esto plantea un reto importante para las agencias reguladoras del medio ambiente, los fabricantes de automotrices y la industria del combustible.

\subsubsection{Compuestos oxigenados}

El octanaje de las gasolinas también puede elevarse mediante la adición de compuestos oxigenados como son etanol, isopropanol, metanol y metil-tert-butiléter (Demirbas et al., 2015). La Carta Mundial de Combustibles (WWFC) mantiene que el metanol no debe utilizarse dado su carácter corrosivo, que daña los componentes metálicos del motor y produce la degradación de plásticos y elastómeros (World Wide Fuel Charter, 2013). El etanol es el aditivo oxigenado más empleado por ser más rentable y un buen potenciador del octanaje. Así, por ejemplo, un $10 \%$ de etanol en la gasolina aumenta el RON y el MON en 3.2 y 1.5 puntos, respectivamente (Australian Government, 2000). No obstante, pese a que el etanol aumenta el octanaje de las gasolinas y disminuye las emisiones de monóxido de carbono, también incrementa la volatilidad del combustible y, por lo tanto, aumenta las emisiones por evaporación. Se ha visto, por ejemplo, que empleando una gasolina con un $10 \%$ de 
etanol las emisiones por evaporación aumentan en un $12 \%$ en comparación con una gasolina con hidrocarburos como únicos componentes. Esto puede deberse en parte a la permeación de combustible a través de los elastómeros que forman gomas y partes plásticas del sistema de conducción de combustible y sus vapores (World Wide Fuel Charter, 2013). Por otra parte, el etanol es totalmente miscible con el agua que, en pequeńa cantidad, suele estar presente en el combustible, pudiendo inducir a la separación de fases (Australian Government, 2000). Brasil ha sido un país pionero en este sentido, dado que en 1975 comenzó con el primer programa de utilización del etanol como combustibles (ProAlcool), seguido de Estados Unidos en 1978 (Wheals et al., 1999), en donde la mezcla de gasolina con un $10 \%$ de etanol (E10) se emplea habitualmente.

El metil-tert-butiléter (MTBE) es frecuentemente utilizado como aditivo en las gasolinas desde 1973. Un $10 \%$ (v/v) de este compuesto aumenta el octanaje de las gasolinas en una cantidad comprendida entre 1.5 y 3 puntos, lo que permite reducir el contenido en hidrocarburos aromáticos (Palencia, Folgeras y Gómez, 2014). No obstante, como sucede con otros éteres, el MTBE es volátil (temperatura de ebullición: $55.3^{\circ} \mathrm{C}$ ) y pasa a la atmósfera rápidamente. Por otra parte, dado su solubilidad en agua ( $42 \mathrm{~g} / \mathrm{L}$ a $20^{\circ} \mathrm{C}$ ), persistencia y movilidad, puede contaminar los acuíferos (Trapp, Yu \& Mosbaek, 2003; Songolzadeh, Soleimani \& Behnood, 2013). La contaminación por MTBE es considerada un problema ambiental crítico y se cree que puede ser peligrosa para la salud humana. Así, por ejemplo, la exposición aguda al MTBE puede producir dolor de cabeza, irritación en nariz, garganta y ojos. Además, algunos estudios animales le atribuyen un carácter carcinogénico (Salimi et al., 2016). La Carta Mundial de Combustibles establece para sus cinco categorías de gasolinas un contenido máximo de oxígeno de $2.7 \%$ (p/p), valor coincidente con la directiva 98/70/EC de la Unión Europea (World Wide Fuel Charter, 2013). Las especificaciones europeas permiten un máximo de $15 \%$ (v/v) de éteres de cinco o más carbonos en las gasolinas (Directiva98/70/CE del Parlamento Europeo, 1998; Australian Government, 2014). 


\subsubsection{Alquenos}

Los alquenos u olefinas son hidrocarburos insaturados presentes en las gasolinas en baja proporción, aunque los procesos de craqueo aumentan su concentración en las mismas. Un caso interesante son los crudos del Pennsylvania, en lo que se ha encontrado cantidades importantes de este tipo de compuestos (Curiale \& Frolov, 1998). Generalmente, los alquenos tienen un índice de octanos superior al de los alcanos y por ello mejoran el octanaje de la gasolina (Ramadhan, Al-Hyali, 1999). En la tabla 1 se pueden observar dos casos llamativos. Por ejemplo, cuando se introduce una insaturación en la cadena de heptano, su AKI pasa de valor cero a un valor de entre 71.1 y 84.7, dependiendo de en qué posición esté el doble enlace (entradas 1, 5 y 6). En el caso del octano, sucede algo parecido. El AKI pasa de un valor de cero a otro de 87.6, cuando se introduce una insaturación en el carbono 4 (entradas 2 y 8). No obstante, dada su inestabilidad térmica, las olefinas pueden reaccionar con el oxígeno de la atmósfera. Los productos de oxidación son sólidos insolubles que se adhiere a las superficies metálicas del motor formando depósitos desde el tanque de combustible hasta la cámara de combustión. La acumulación de estos productos puede causar desgaste del motor y puede tener efectos adversos sobre las prestaciones, emisiones y durabilidad del mismo. Además, tras su evaporación a la atmósfera, las olefinas inducen a la formación de ozono, que resulta tóxico (US-EPA). Por otra parte, sus productos de combustión forman dienos tóxicos (Pradelle, Braga, Martins, Turkovics y Pradelle, 2015). En este sentido, resulta especialmente tóxico el 1,3-butadieno, un conocido carcinógeno que se forma durante la combustión de los alquenos en los motores de gasolina (Australian Government, 2000). Por todo lo anterior, el contenido en alquenos también está limitado en las normativas avanzadas. Así, por ejemplo, el contenido en alquenos en la Unión Europea fue limitado al 18 \% v/v. El Estado de California permite un máximo (plano) del $6 \% \mathrm{v} / \mathrm{v}$ o una media del $4 \% \mathrm{v} / \mathrm{v}$ con máximos del $10 \%$ v/v (Kirchstetter et al., 1999; Fung, 2011).

\subsubsection{Compuestos sulfurados}

Los compuestos sulfurados son componentes normales del petróleo, donde varían desde el 0.05 hasta más del $14 \%$ en peso, aunque pocos crudos producidos comercialmente exceden $4 \%$ de azufre, siendo el 
contenido promedio del $0.65 \%$. La cantidad de azufre presente permite clasificar a los crudos en dulces $(<0.5 \%$ de azufre) y agrios $(>1 \%$ de azufre). Estos compuestos, que incluyen sulfuro de hidrógeno, mercaptanos o tioles, sulfuros, disulfuros, tiofenos, benzotiofenos, dibenzotiofenos, benzonaftotiofenos y dinaftotiofenos, provienen en su mayoría de una fuente orgánica (tabla 2) (Orr y Damste, 1990).

Tabla 2. Ejemplo de algunos compuestos sulfurados encontrados en el petróleo

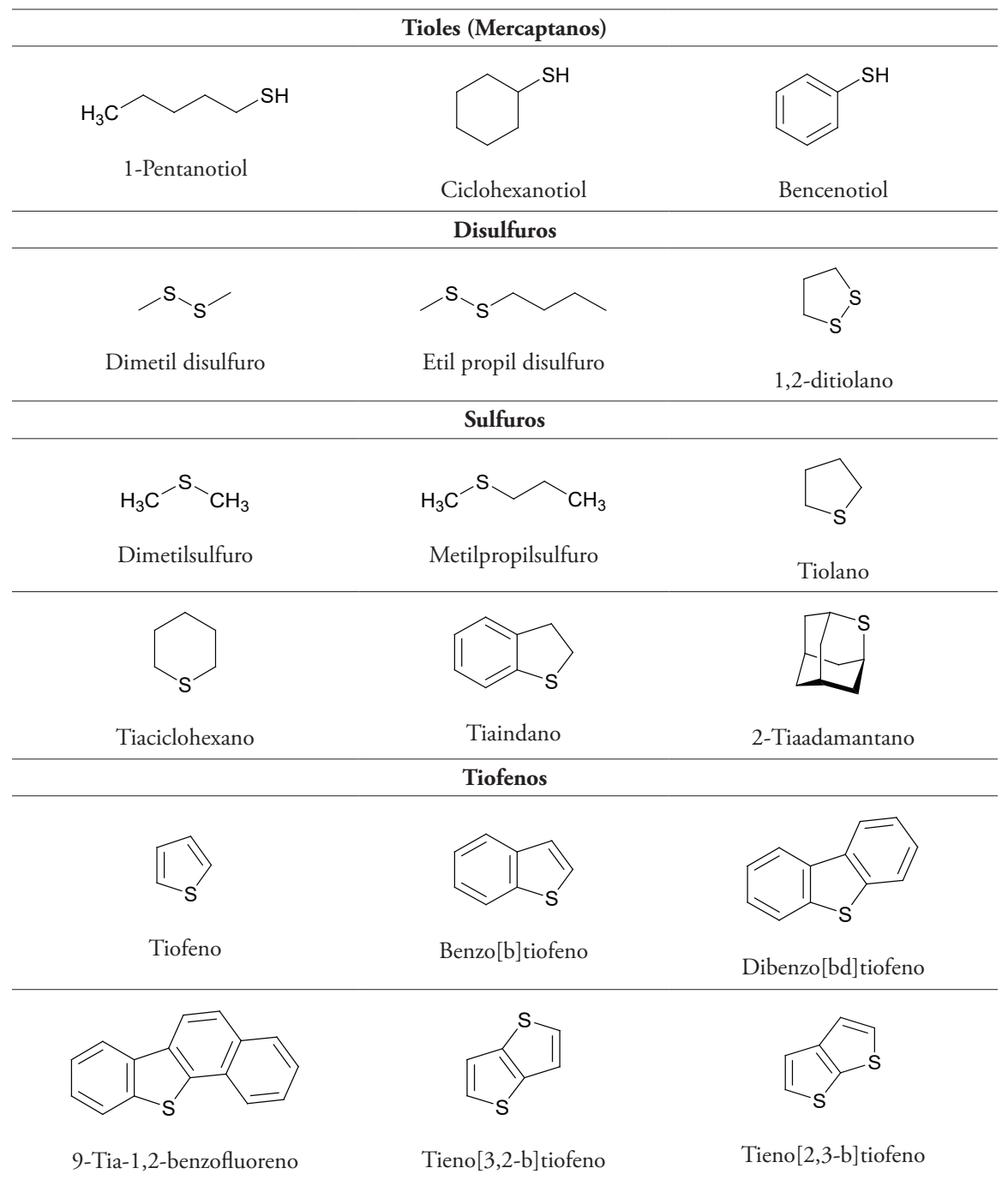


Los compuestos sulfurados son eliminados en mayor o menor medida durante el proceso de refinado, ya que su presencia en las gasolinas reduce la eficacia de los catalizadores de los vehículos que limitan las emisiones de los vehículos (Demirbas et al., 2015). Mantener unos bajos niveles de azufre en las gasolinas es necesario para reducir las emisiones de óxidos de azufre, en los que se transforma cualequier componente sulfurado presente en el combustible. Estos óxidos son dañinos para el medio ambiente por formación de lluvia ácida. La eliminación del azufre se realiza por un proceso llamado hidrodesulfuración, en el que la fracción hidrocarbúrica es mezclada con hidrógeno y pasada por un catalizador de hidrodesulfuración bajo adecuadas condiciones de presión y temperatura. Los catalizadores formados por compuestos de níquel-cobalto-molibdeno o de níquel-molibdeno con soporte de alúmina son los más eficaces. El objetivo de este proceso es romper los enlaces de carbono-azufre presentes en el material a tratar y saturar con hidrógeno las valencias libres resultantes o los dobles enlaces olefínicos formados durante el proceso, convirtiendo tanto como sea posible del contenido de azufre orgánico en hidrocarburos y en ácido sulfhídrico (Dhar et al., 2005).

\section{Obtención de gasolinas}

Las gasolinas pueden obtenerse por destilación fraccionada del petróleo. Tras la destilación de los elementos más volátiles del crudo (hasta unos $20^{\circ} \mathrm{C}$ ) que rinde metano, etano, propano, butano e isobutano, se obtiene la nafta ligera $\left(20^{\circ} \mathrm{C}-150^{\circ} \mathrm{C}\right)$. Esta segunda fracción, conocida también como gasolina ligera o ligroína, puede ser sometida a destilación para obtener una mezcla ligera de hidrocarburos conocida como éter de petróleo, de la que luego se puede obtener, mediante redestilación, entre $55^{\circ} \mathrm{C}$ y $65^{\circ} \mathrm{C}$ el hexano comercial, muy utilizado como disolvente en química orgánica y en la industria de los aceites. La fracción restante de nafta ligera se utiliza para la obtención de gasolinas, aunque su bajo octanaje (en torno a 70 octanos) precisa de los procesos de craqueo y reformado catalítico para elevar su capacidad antidetonante hasta los valores comerciales (US-EPA, 2008). Una tercera fracción de destilación $\left(150-200^{\circ} \mathrm{C}\right)$ rinde la nafta pesada, que debe ser sometida a reformado catalítico que modifica su composición y eleva su octanaje (US-EPA, 2008). Estas son las llamadas 
gasolinas de destilación directa, pero estos combustibles también pueden obtenerse a partir de fracciones pesadas del petróleo mediante el craqueo térmico o catalítico de hidrocarburos de mayor peso molecular. Esto último es especialmente relevante si tenemos en cuenta que la demanda de gasolina del mercado es mucho mayor que el rendimiento del petróleo en este combustible, que suele oscilar entre el 10 y $30 \%$. Por esta razón se recurre a procesos que permiten obtener hidrocarburos con cadenas carbonadas en el intervalo de entre 5-10 carbonos, que están en el rango de hidrocarburos integrantes de las gasolinas (Li et al., 2007).

\subsection{Craqueo térmico}

Este proceso desarrollado en torno a 1913 y conocido también como pirólisis, consiste en someter a las parafinas pesadas a elevadas temperaturas $\left(500-700^{\circ} \mathrm{C}\right.$ ) y presiones (alrededor de $20 \mathrm{~kg} / \mathrm{cm}^{2}$ ) en ausencia de oxígeno para prevenir su combustión, y así provocar su fragmentación a través de reacciones radicalarias que conducen finalmente a alcanos más pequeños y alquenos (Sadrameli, 2015). Este proceso permite incrementar el rendimiento en la producción de gasolina de un $40 \%$ y alcanzar valores de octanaje en torno a 75 (Schobert, 1990).

En cuanto al mecanismo de reacción, el craqueo térmico se realiza mediante un proceso radicalario, en el que se pueden distinguir las tres etapas características de este proceso (Figura 1) (Mengual, 2009).

En la etapa de iniciación, la ruptura homolítica, principalmente, de un enlace carbono-carbono de la parafina conduce a radicales libres. Una vez formados, y debido a su alta reactividad, reaccionan de diferentes formas en la etapa de propagación, dando lugar a productos más estables. Esta propagación es clave para entender la distribución de productos obtenidos en el proceso. Podemos distinguir las siguientes reacciones:

- Reacciones de activación o de abstracción de hidrógeno (figura 1, sección b.1), en las que los intermedios tipo radical libre toman un átomo de hidrógeno de una de sus moléculas vecinas, originando un nuevo radical. Estos procesos están favorecidos cuando 
el radical formado es mucho más estable que el inicial. Se observa experimentalmente que los radicales terciarios son más estables que los secundarios y estos a su vez más estables que los primarios. El fenómeno que estabiliza los radicales libres se conoce como hiperconjugación.

- Las reacciones de $\beta$-escisión son otra forma en que puede producirse el acortamiento de la cadena de los hidrocarburos durante el craqueo térmico (figura 1 , sección b.2). La reacción comienza un radical libre en el que se rompe un enlace carbono-carbono en posición $\beta$ al mismo, originando la mezcla de una olefina y otro radical libre, que pude sufrir de nuevo escisión $\beta$. Este tipo de reacciones son las responsables de la formación de olefinas en los procesos de craqueo térmico. Es importante señalar que la reacción de polimerización, que se puede considerar como inversa a la $\beta$-escisión, ocurre marginalmente debido a las elevadas temperaturas del proceso.

- Las reacciones de isomerización (figura 1, sección b.3) se originan en aquellos radicales libres que son lo suficientemente largos para adoptar una conformación de semisilla, produciéndose una abstracción de hidrógeno intramolecular, preferentemente a partir de la posición 5 , originando radicales secundarios, que pueden sufrir seguidamente las reacciones de fisión.

Finalmente, en la etapa de terminación, los radicales sufren reacciones que los neutralizan produciendo especies inertes. Aunque existen diversas formas de terminación del proceso, la más común es cuando se encuentran dos radicales. Esta reacción bimolecular puede seguir dos caminos:

- Terminación por combinación (figura 1 , sección c.1), la cual da como resultado la formación de un alcano con un tamańo igual a la suma de los radicales que se unieron. Estas reacciones están favorecidas tanto desde el punto de vista cinético como termodinámico. No obstante, debido a la baja concentración relativa de los radicales, es más probable que estos reaccionen con otras moléculas de naturaleza no radicalaria. 
- Terminación por desproporción (figura 1, sección c.2), que da como resultado dos moléculas, una saturada y otra insaturada. Esta es la razón por la que aparecen olefinas en las gasolinas, y dado que los alquenos tienen mayor índice de octanos que los alcanos, la presencia de estos en el combustible, procedentes del craqueo térmico, ayuda a mejorar las propiedades antidetonantes del mismo.<smiles>[R]CCCCC([R])C([R])[R]</smiles><smiles>[R]C([R])C([R])CCCC</smiles>

\section{b) ETAPA DE PROPAGACIÓN}

b.1 Reacciones de activación o de abstracción de hidrógeno
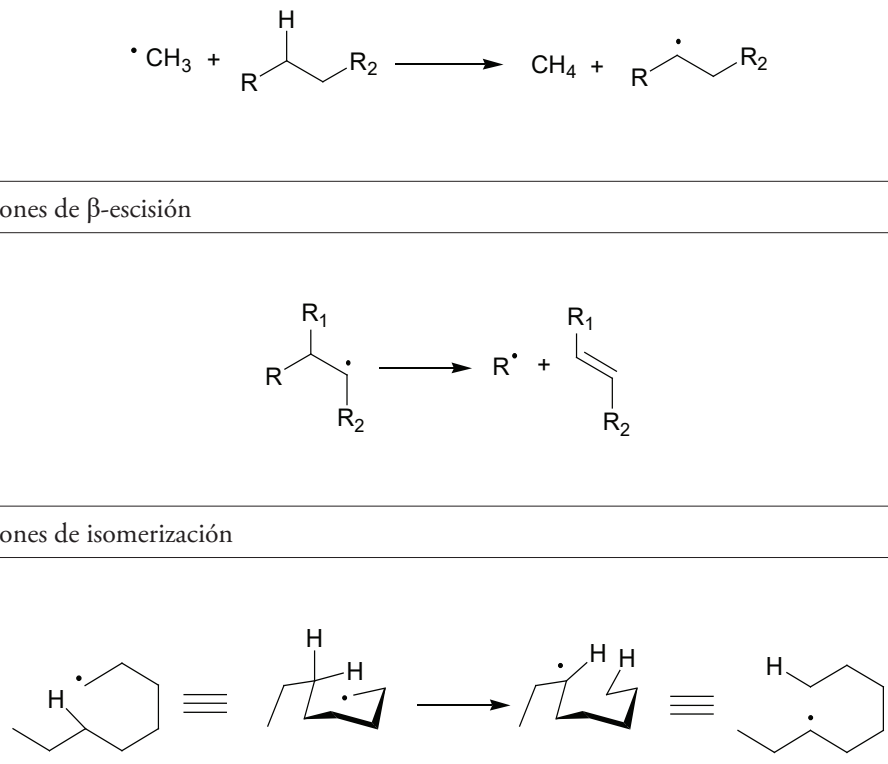


\section{c) ETAPA DE TERMINACIÓN}

c.1 Reacciones de combinación<smiles>[R2]CC([R])[CH-][CH]C</smiles><smiles>[R]CC([R])C([R])C([R])CC</smiles>

c.2 Reacciones de desproporción

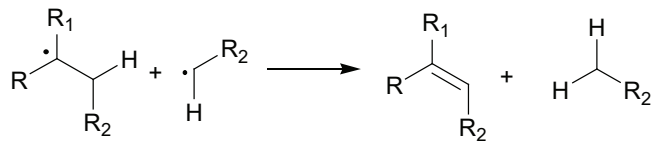

Figura 1: Reacciones típicas del craqueo térmico

Las reacciones de recombinación que experimentan los radicales secundarios durante el craqueo térmico producen una proporción mayor de hidrocarburos ramificados que mejoran el índice de octanos de las gasolinas hasta un valor de aproximadamente 75. No obstante, este es un valor insuficiente para satisfacer la demanda de octanaje de los fabricantes de muchos motores, que por su relación de compresión, requieren una gasolina de mayor octanaje. Por ello se dice que las gasolinas producidas mediante este proceso son relativamente de baja calidad, por lo tanto se requieren otros procesos que permite elevar la proporción de isoparafinas en las gasolinas o utilizar aditivos que eleven el índice de octanos (Vogt \& Weckhuysen, 2015).

\subsection{Craqueo catalítico}

El craqueo catalítico es el proceso de refinado, desde el origen, más importante en la destilación del crudo. Cuando los hidrocarburos de 
elevado peso molecular son calentados en presencia de un catalizador y en ausencia de oxígeno, estos se fragmentan en compuestos relativamente cortos $\left(\mathrm{C}_{5}-\mathrm{C}_{10}\right)$. La presencia del catalizador permite que las reacciones producidas sean mucho más rápidas y selectivas, y que se produzcan a una temperatura y a una presión $\left(1-1.5 \mathrm{~kg} / \mathrm{cm}^{2}\right.$ frente a los $\left.20 \mathrm{~kg} / \mathrm{cm}^{2}\right)$ menores a las empleadas en el craqueo térmico, produciéndose una gasolina de alta calidad, que puede alcanzar un octanaje de 100 , en un $50 \%$ de rendimiento. Sin embargo, las reacciones generan una cantidad de carbón que se deposita sobre la superficie del catalizador, por lo que los procesos se desarrollan en forma continua, mediante una circulación de catalizador que se contacta íntimamente con la carga. Posteriormente, el catalizador se regenera por medio de la combustión del carbón producido, lo que origina la energía que requiere el sistema para funcionar.

Mientras que el craqueo térmico transcurre a través de mecanismos radicalarios, el craqueo catalítico tiene lugar a través de mecanismos iónicos en los que se forman carbocationes. Al contrario de lo que sucede con los radicales, los carbocationes típicamente sufren procesos de reordenamiento por migración de hidruros y grupos alcanuro, que tiene como consecuencia la formación de alcanos ramificados a partir de alcanos lineales (Schobert, 1990), que tienen una incidencia positiva en el índice de octanos de las gasolinas, aumentando la relación MON/RON (Demirbas et al., 2015). La formación de carbocationes es posible debido a que los catalizadores empleados actualmente son zeolitas (aluminosilicatos como la zeolita Y estabilizada con tierras raras o la zeolita ZSM-5) que se comportan como ácidos de Lewis, promoviendo la ruptura heterolítica de los enlaces C-H. Tal como se muestra en la figura 1, la abstracción de un hidruro por parte del catalizador requiere de este un fuerte carácter electrofílico. El carbocatión secundario formado de esta manera sufre espontáneamente un reordenamiento para formar un carbocatión más estable, que tras aceptar un hidruro da lugar a un alcano ramificado con una capacidad antidetonante mayor que el alcano de partida (Vogt \& Weckhuysen, 2015). 


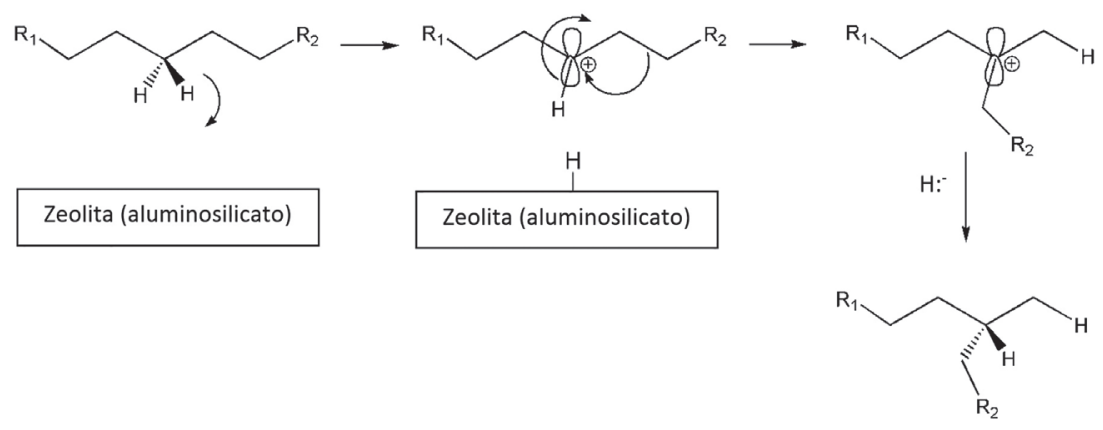

Figura 2. Abstracción de un hidruro a un alcano por parte del catalizador (zeolita) durante el craqueo catalítico, reordenamiento del carbocatión resultante y captura de un hidruro para forma un alcano ramificado con mayor resistencia a la autoignición que el alcano de partida.

\subsection{Reformado catalítico}

El reformado catalítico permite convertir alcanos lineales de bajo octanaje en cicloalcanos y compuestos aromáticos, para ello se utilizan altas temperaturas $\left(490-530{ }^{\circ} \mathrm{C}\right)$ y presiones moderadas (10-25 bar), produciéndose como subproducto valioso hidrógeno (Demirbas et al., 2015). La transformación de parafinas en cicloparafinas o hidrocarburos aromáticos incrementa de manera importante el octanaje como ocurre, por ejemplo, en la conversión del $n$-hexano $(\mathrm{RON}=24.8)$ en ciclohexano $(\mathrm{RON}=84.0)$ o en benceno ( $\mathrm{RON}=90.0)$ mediante deshidrociclación $[15,16]$. Los catalizadores empleados en el reformado catalítico emplean un óxido como $\mathrm{Al}_{2} \mathrm{O}_{3}$ y un metal de transición como $\mathrm{Pd}, \mathrm{Pt}, \mathrm{Ni}, \mathrm{Ir}, \mathrm{Ru}$ y $\mathrm{Rh}$, aunque habitualmente se emplean Pd, Pt o Ni (Nabgan et al., 2018).

Este proceso se lleva a cabo en unidades diseñadas al efecto y que con frecuencia adoptan nombres registrados. El más extendido es el de Platformado, cuya licencia pertenece a UOP (Universal Oil Products), empresa estadounidense que empezó a comercializarla en 1949. Otras empresas de ingeniería también licencian este proceso (p. ej. el antiguo Instituto Francés del Petróleo, que ahora se llama Axens) y algunas de las grandes empresas del petróleo disponen de diseños propios. 


\section{Normativas reguladoras}

Las diferentes normativas que regulan la composición de las gasolinas en las diferentes naciones, recogen los valores máximos y/o mínimos de determinados parámetros físico-químicos como son la densidad, la presión de vapor, la curva de destilación o el índice de octanos, que se relacionan principalmente con las especificaciones técnicas indicadas por los fabricantes de la industria automovilísitca. Por otra parte, estas normativas también limitan la cantidad de componentes de los combustibles que pueden generar emisiones tóxicas, si bien hay diferencias importantes entre los países desarrollados y aquellos que están en vías de desarrollo (Rodríguez, 2012). En este sentido, cabe destacar, además de la presencia de óxidos de azufre en los gases de combustión de los combustibles hidrocarbúricos, los hidrocarburos aromáticos que se cuentan típicamente entre otros componentes ambientalmente perjudiciales de tales gases de combustión. Estos pueden estar presentes debido a una combustión incompleta. Por otra parte, también hay que mencionar la emisión de partículas carbonosas que a menudo contienen hidrocarburos aromáticos policíclicos, compuestos metálicos, materiales orgánicos oxigenados y otros materiales potencialmente tóxicos.

Debido a la creciente preocupación actual en torno a la polución ambiental, están siendo desarrolladas en todo el mundo diversas legislaciones nacionales que imponen límites cada vez más rigurosos a los niveles permitidos de impurezas y sustancias potencialmente tóxicas en los combustibles hidrocarbúricos. Un buen ejemplo de esto es la drástica reducción impuesta al contenido en azufre del gasóleo de automoción por Estados Unidos, desde niveles permitidos de 1000 ppm en 1990, hasta 15 ppm en 2006, tendencia que ha sido seguida por la Unión Europea. No obstante, entre los países en vías de desarrollo es posible encontrar límites admisibles para el contenido en azufre del gasóleo en torno a los $5000 \mathrm{ppm}$, lo que afecta muy negativamente tanto a las emisiones como a la duración del motor de los automóviles (World Wide Fuel Charter, 2013; Australian Government, 2014; Rodríguez, 2012). 
La Agencia para la Protección del Medio Ambiente de Estados Unidos (United States Environmental Protection Agency) ha propuesto recientemente reglamentos que limitarían el contenido de azufre al $0.05 \%$ en peso y el contenido de aromáticos al $20 \%$ volumétrico en los combustibles Diesel para el tráfico en carretera. Tales reglamentos exigen a las refinerías afrontar adicionales exigencias en materia del tratamiento de los combustibles Diesel, y mayores costes de inversión y explotación. Analizando esta tendencia, no deben descartarse reducciones adicionales de los niveles admisibles del contenido de estas sustancias tóxicas pese a los retos que el problema plantea. Esto es especialmente importante en aquellos países que no cuentan aún con normativas avanzadas que impongan límites lo suficientemente estrictos, como para asegurar la preservación de la salud de la población y el medio ambiente.

\section{Conclusión}

Las gasolinas son mezclas complejas formadas principalmente por hidrocarburos que exhiben una notable diversidad estructural, siendo algunos de ellos tóxicos. La composición exacta de una gasolina va a depender de factores como el petróleo del cual se ha obtenido, los procesos de obtención y procesamiento, como el craqueo o el reformado catalítico, y también de las normativas reguladoras. Estas determinan los valores correctos de aquellas especificaciones que son esenciales para asegurar el buen funcionamiento de los motores y a la vez limitan el contenido de aquellos compuestos considerados tóxicos. En este sentido, se pone de manifiesto la necesidad de disponer de normativas avanzadas, actualizadas y homogéneas que estén en consonancia con las evidencias científicas que muestran que algunos de los componentes de las gasolinas resultan perjudiciales para la salud y el medio ambiente.

\section{Referencias}

Australian Government. (2000). Department of the Environment and Energy. Environment Australia (National Heritage Trust), Setting National Fuel Quality Standards - Paper 2- Proposed Standards for Fuel Parameters (Petrol and Diesel), Canberra, Australia. 
Australian Government. (2014). Department of the Environment and Energy. International fuel quality standards and their implications for australian standards. Final report. Hartenergy Research \& Consulting. Disponible en https:/www.environment.gov.au/system/files/ resources/f83ff2dc-87a7-4cf9-ab24-6c25f2713f9e/files/international-feul-quality-standards.pdf [acceso 15 de abril 2019].

Brudzewski, K., Kesik, A., Kołodziejczyk, K., Zborowska, U. \& Ulaczyk, J. (2006). Gasoline quality prediction using gas chromatography and FTIR spectroscopy: An artificial intelligence approach, Fuel, 85(4), 553-558.

Curiale, J.A. \& Frolov, E.B. (1998). Occurrence and origin of olefins in crude oils. A critical review. Organic Geochemistry, 29, 397-408.

Dhar, G.M., Kumaran, G.M., Kumar, M., Rawat, K.S., Sharma, L.D., Raju, B.D. \& Rao, K.S.R. (2005). Physico-chemical characterization and catalysis on SBA-15 supported molybdenum hydrotreating catalysts, Catalysis Today, 99, 309-314.

Demirbas, A., Balubaid, M.A., Basahel, A.M., Ahmad, W. \& Sheikh, M.H. (2015). Octane rating of gasoline and octane booster additives. Petroleum Science and Technology, 33, 1190-1197.

Directiva98/70/CE del Parlamento Europeo y del Consejo de 13 de octubre de 1998 relativa a la calidad de la gasolina y el gasóleo y por la que se modifica la Directiva 93/12/CEE del Consejo. Disponible en: https://eur-lex.europa.eu/LexUriServ/LexUriServ.do?uri=CONSLEG: 1998L0070:20031120:ES:PDF

Donald, J.M., Hooper, K. \& Hopenhayn-Rich, C. (1991). Perspectives reproductive and developmental toxicity of toluene: a review, Environmental Health, 94, 237-244.

Durbin, T.D., Miller, J.W., Younglove, T., Huai, T. \& Cocker, K. (2007). Effects of fuel ethanol content and volatility on regulated and unregulated exhaust emissions for the latest technology gasoline vehicles. Environmental Science \& Technology, 41, 4059-4064.

Fung, F. (2011). Best Practices for Fuel Quality Inspection Programs. International Council on Clean Transportation. Working paper No. 2011-11, Washington, November, 2011. [acceso 15 de abril 2019]. Disponible en: https://www.theicct.org/sites/default/files/publications/ICCT_fuelqualityBP_nov2011.pdf 
Gary, J.H. \& Handwerk, G.E. (1980). Refino del petróleo: tecnología y economía. Editorial Reverté. 37-186. Barcelona, España.

Hajbabaei, M., Karavalakis, G., Miller, W.J., Villela, M., Huaying, X.K. $\&$ Durbin T.D. (2013). Impact of olefin content of criteria and toxic emissions from modern gasoline vehicles, Fuel, 107, 671-679.

Harley, R.A., Hooper, D.S., Kean, A.J., Kirchstetter, T.W., Hesson, J.M., Balberan, N.T., Stevenson, E.D. \& Kendall, G.R. (2006). Effects of reformulated gasoline and motor vehicle fleet turnover on emissions and ambient concentrations of benzene. Environmental Science \& Technology, 40, 5084-5088.

Karavalakis, G., Short, D., Vu, D., Russell, R., Hajbabaei, M., Asa-Awuku, A. \& Durbin, T.D. (2015). Evaluating the effects of aromatics content in gasoline on gaseous and particulate matter emissions from SI-PFI and SIDI vehicle. Environmental Science \& Technology, 49, 7021-7031.

Khalade, A., Jaakkola, M.S., Pukkala, E. \& Jaakkola, J.J.K. (2010). Exposure to benzene at work and the risk of leukemia: a systematic review and meta-analysis. Environmental Health, 9, (31),1-8. Disponible en: https://ehjournal.biomedcentral.com/track/pdf/ 10.1186/1476-069X-9-31

Kinsara, R.A. \& Demirbas, A. (2016). Upgrading of crude oil via distillation processes. Petroleum Science and Technology, 34(14), 1300-1306.

Kirchstetter, T.W., Singer, B., Harley, R.A., Kendalland, G.R. \& Traverse, M. (1999). Impact of California reformulated gasoline on motor vehicle emissions. 1. Mass emission rates. Environmental Science \& Technology, 33, 318-328.

Lee, D.M., Lee, D.H. \& Hwang, I.H. (2018). Gasoline quality assessment using fast gas chromatography and partial least-squares regression for the detection of adulterated gasoline, Energy Fuels, 32(10), 10556-10562.

Li, S., Zhang, Q., Sun, X., Fan, Q. \& Chen, J. (2007). Distillation yields and properties from blending crude oils: Maxila and cabinda crude oils, maxila and daqing crude oils, Energy Fuels, 21, 1145-1150.

Mengual. J. (2009). Craqueo térmico y catalítico, con y sin vapor de agua, de alcanos sobre zeolitas. Cinética, desactivación y estabilización del 
catalizador, (tesis doctoral). Universitat de Valencia, Valencia, España. Disponible en: tdx.cat/bitstream/handle/10803/31944/ mengual.pdf?sequence $=1$

Nabgan, W., Rashidzadeh, M. \& Nabgan, B. (2018). The catalytic naphtha reforming process: hydrodesulfurization, catalysts and zeoforming, Environmental Chemistry Letters, 16, 507-522.

Orr, W.L. \& Damste, J. S. S. (1990). Geochemistry of sulfur in petroleum systems, En W. L. Orr and C. M. White (ed.), Geochemistry of sulfur in fossil fuels. American Chemical Society, Washington, D.C, p. 2-29.

Palencia, F.D., Folgeras, M.B. \& Gómez, F. (2014). Influencia de los aditivos oxigenados sobre las propiedades de las gasolinas. Universidad de Oviedo, Master Universitario en Ingeniería Energética España. Disponible en: http://digibuo.uniovi.es/dspace/bitstream/10651/27919/3/ TFMFranciscoDanielPalenciaProteg.pdf

Perdih, A. \& Perdih, F. (2006). Chemical interpretation of octane number. Acta Chimica Slovenica. 53, 306-315.

Pradelle, F., Braga, S.L., Martins, A.R.F.A., Turkovics, F. \& Pradelle, R.N.C. (2015). Gum Formation in Gasoline and Its Blends: A Review. Energy Fuels, 29, 7753-7770.

Ramadhan, O.M. \& Al-Hyali, E.A. (1999). New experimental and theoretical relation to determine the research octane number $(\mathrm{RON})$ of authentic aromatic hydrocarbons could be present in the gasoline fraction. Petroleum Science and Technology, 17, 623-635.

Re-Poppi, N., Alemeida, F.F.P., Cardoso, C.A.L., Raposo Jr, J.L., Viana, L.H. \& Silva, T.Q. (2009). Screening analysis of type C Brazilian gasoline by gas chromatography-Flame ionization detector. Fuel, 88, 418-423.

Ritter, S. What's that stuff? Gasoline. (2005). Chemical \& Engineering News, 83(8), 37.

Rodríguez, N. (2012). Análisis de la reducción del azufre en el combustible diesel en El Salvador, Guatemala, Honduras y Nicaragua. Ministerio Federal de Cooperación Económica y desarrollo (Alemania); Comisión Económica para América Latina y el Caribe (CEPAL). Disponible en: https://repositorio.cepal.org/handle/11362/26095 
Sadrameli, S.M. (2015). Thermal/catalytic cracking of hydrocarbons for the production of olefins: A state-of-the-art review I: Thermal cracking review. Fuel, 140, 102-115.

Salimi, A., Vaghar-Moussavi, M., Seydi, E. \& Pourahmad, J. (2016). Toxicity of methyl tertiary-butyl ether on human blood lymphocytes. Environmental Science and Pollution Research, 23, 8556-8564.

Schifter, I., Díaz, L., Gonzalez, U. \& González-Macias, C. (2013). Fuel formulation for recent model light duty vehicles in Mexico base on a model for predicting gasoline emissions. Fuel, 107, 371-381.

Schobert, H.H. (1990). The Chemistry of Hydrocarbon Fuels ( $1^{\text {st }}$ ed.). Londres, Inglaterra: Butterworth \& Co (Publishers), 58-63, 184-196, 211-246.

Songolzadeh, M., Soleimani, M. \& Behnood, R. (2013). A brief review of methyl tert-butyl ether (MTBE) removal from contaminated air and water. Research Journal Chemistry Environment, 17, 90-97.

Swick, D., Jaques, A., Walker, J.C. \& Estreicher, H. (2014). Gasoline risk management: A compendium of regulations, standards, and industry practices. Regulatory Toxicology and Pharmacology, 70, 80-92.

Trapp, S., Yu, X. \& Mosbaek, X. H. (2003). Persistence of methyl tertiary butyl ether (MTBE) against metabolism by danish vegetation. Environmental Science and Pollution Research, 10, 357-360.

United States Environmental Protection Agency (US-EPA). (2017). Health Effects of Ozone in the General Population. Disponible en: https://www.epa.gov/ozone-pollution-and-your-patients-health/ health-effects-ozone-general-population

United States Environmental Protection Agency (US-EPA). (2008). $A P$ 42, ( $5^{\text {th }}$ Edition), Volume I. Chapter 5: Petroleum Industry, Transportation and marketing of Petroleum Liquids. Disponible en: www.epa.gov/ttn/chief/ap42/ch05/final/c05s02.pdf

Vempatapu, B.P. \& Kanaujia, P.K. (2017). Monitoring petroleum fuel adulteration: A review of analytical methods, Trends in Analytical Chemistry, 92, 1-11.

Verma, DK., des Tombe, K. (2002). Benzene in gasoline and crude oil: occupational and environmental implications. AIHA Journal. 63, 225-230. 
Vogt, E.T.C., Weckhuysen, B. M (2015). Fluid catalytic cracking: recent developments on the grand old lady of zeolite catalysis. Chemical Society Reviews, 44, 7342-7370.

Von Euler, M., Phama, M.T., Hillefors, Mi., Börje, B., Henriksson, B. \& Von Euler, G. (2000). Inhalation of low concentrations of toluene induces persistent effects on a learning retention task, beam-walk performance, and cerebrocortical size in the rat. Experimental Neurology, 163, 1-8.

Wheals, A. E., Basso, L. C., Alves, D. M. G. \& Amorim. H.V. (1999). Fuel ethanol after 25 years. Trends in Biothecnology, 17, 482-487.

Wiedemann, L.S.M., d'Avila, L.A. \& Azevedo, D. A. (2005). Brazilian gasoline quality: Study of adulteration by statistical and gas chromatography. Journal of the Brazilian Chemical Society, 16(2), 139-146.

World Wide Fuel Charter. (2013). European Automobile Manufacturers Association (ACEA) \& Alliance of Automobile Manufacturers, Truck and Engine Manufacturers Association (EMA) \& Japan Automobile Manufacturers Association (JAMA). ( $5^{\text {th }}$ Edition). Disponible en: https://www.acea.be/uploads/publications/Worldwide_Fuel_Charter_5ed_2013.pdf_

Yang, M., Kim, S. H., Kim, J. C., Shin, T. \& Moon, C. (2010). Toluene induces depression-like behaviors in adult mice. Toxicological Research, 26, 315-320.

Yao, Y. C. \& Tsai, J. H. (2013). Effects of gasoline aromatic content on emissions of volatile organic compounds and aldehydes from a fourstroke motorcycle. Environmental Technology, 34, 2531-2539.

Yardley-Jones, A., Anderson, D. \& Parke, D. V. (1991). The toxicity of benzene and its metabolism and molecular pathology in human risk assessment. The British Journal of Industrial Medicine, 48, 437-444. 